\title{
Prolonged Storage of Red Blood Cells for Transfusion in Citrate Phosphate Dextrose Adenine-1 Affects Their Viability
}

\author{
Samuel Antwi-Baffour1 ${ }^{*}$, Samuel Appiah Danso², Jonathan Adjei', Ransford Kyeremeh1, \\ Michael Mark Addae ${ }^{1}$ \\ ${ }^{1}$ Department of Medical Laboratory Sciences, School of Allied Health Sciences, College of Health Sciences, \\ University of Ghana, Accra, Ghana \\ ${ }^{2}$ The Accra Area Blood Collection Site of the Southern Area Blood Centre (SABC), the Korle-Bu Teaching \\ Hospital, Accra, Ghana \\ Email: s.antwi-baffour@chs.edu.gh, appiahdanso2004@yahoo.com, jonathanadjei@gmail.com, \\ rkyeremeh@gmail.com, mmaddae@chs.edu.gh
}

Received 3 September 2015; accepted 18 September 2015; published 25 September 2015

Copyright (C) 2015 by authors and OALib.

This work is licensed under the Creative Commons Attribution International License (CC BY). http://creativecommons.org/licenses/by/4.0/

(c) (i) Open Access

\section{Abstract}

Most donor blood units for transfusion purposes are stored in Citrate Phosphate Dextrose Adenine-1 (CPDA-1) at blood-banks until used. Despite improvements made in blood storage attempts, CPDA-1 may still cause morphological and degenerative changes in red blood cells (RBCs) if storage is prolonged. The purpose of the study was to assess the viability of RBCs through characteristic changes resulting from prolonged storage in CPDA-1. A total of fifteen whole blood stored in CPDA-1 were analyzed for changes in erythrocytes morphology, Packed Cell Volume (PCV) and osmotic fragility in a seven day interval over a thirty-five day period. On day 1 (control), samples were analyzed within 6 hours of collection, and subsequently taken to the blood bank to be stored and re-analyzed from days 7 to 35. Mean Cell Fragility (MCF) which is suggestive of RBC membrane instability showed changes that were statistically significant between days 1 and 35. Morphological changes observed over the storage period included spherocytosis, echinocytosis, and marked sphero-echinocytosis with increased rouleaux formation. PCV value decreased marginally over the period. The author concluded that donor blood units stored for longer periods may result in RBC defects, affecting their viability and consequently interfere with expected therapeutic outcomes.

\section{Keywords}

Blood, Transfusion, Membrane, Red Blood Cells, Morphological, CPDA-1

\footnotetext{
"Corresponding author.
} 


\section{Introduction}

Blood transfusion is generally the process of infusing blood components into one's circulation intravenously. Transfusion therefore is used in a variety of medical conditions to replace lost blood as discussed by Chessbrough [1]. Major surgical operation, accidents resulting in considerable blood loss, cancer patients requiring therapy, women in childbirth, newborn babies in certain cases, patients of hereditary disorders like haemophilia and thalassaemia and severe burn victims are indicators of blood use as discussed elsewhere [2]. A number of the indicator conditions require urgent need for transfusion in order to protect lives. This therefore justifies the need for blood and blood components to be stored under standard blood bank conditions to enable easy access to them in a timely manner as discussed by Hess et al. [3].

The idea of blood storage has in recent times witnessed increased scrutiny regarding efficacy and risk of transfusion associated reaction or events as discussed elsewhere [4]. A variety of changes have been identified within the red blood cells (RBCs) and the storage media during RBCs preservation that are correlated with reduced tissue oxygenation and transfusion-associated adverse effects as discussed elsewhere [5] [6]. These alterations which are collectively termed as storage lesion include extensive biochemical, biomechanical and immunological changes involving cells of diverse origin as discussed by Daryl et al. [5]. Most blood transfusion centers in developing countries are yet to fully adopt components transfusion therapy and therefore rely extensively on whole blood and RBCs for transfusion as discussed elsewhere [4]. The phenomenon requires that a high percentage of viable RBCs must be recovered following storage to achieve efficacious blood transfusion therapy as discussed by Chessbrough [1]. The length of time that blood and blood components are stored before they are transfused is therefore of paramount importance.

Conditions under which blood is stored, in most storage facilities (blood banks) invariably, produces changes in RBCs irrespective of the preservative or anticoagulant used as discussed by Gupta [7]. Some types of anticoagulants may however facilitate deterioration if storage is prolonged. The physical and chemical changes imparted as a result of storage affects red blood cell viability as discussed by Daryl et al. [5]. Due to these changes, therapeutic efficacy expected to be gained after transfusion, to a large extent may be compromised as discussed by Tinmouth and Chin-Yee [8].

One of the commonly used anticoagulant for blood storage is Citrate Phosphate Dextrose Adenine-1 (CPDA1). CPDA-1 solution contains 2 g Dextrose (monohydrate), 1.66 g Sodium Citrate (dihydrate), $188 \mathrm{mg}$ Citric Acid (anhydrous), $140 \mathrm{mg}$ Monobasic Sodium Phosphate (monohydrate) and $17.3 \mathrm{mg}$ Adenine. CPDA-1 is the only anticoagulant-nutrient solution in regular use. Citrate CPDA-1 is used as an anticoagulant for whole blood and is supplied as a high quality, convenient, $0.2 \mu \mathrm{m}$-filtered ready to use solution. Recommended anticoagulant: whole blood ratio is 1:9. The function of CPDA-1 is whole blood preservation to provide viable and functional blood components for patients requiring blood transfusion. More than $70 \%$ of red cells are expected to remain viable in circulation 24 hours after transfusion of blood stored at $2^{\circ} \mathrm{C}-6^{\circ} \mathrm{C}$ in CPDA-1 for 35 days. Knowledge on prolonged storage effects on RBCs by certain anticoagulants will help improve $\mathrm{RBC}$ storage by reducing storage alterations (lesions) that will hitherto affect $\mathrm{RBC}$ viability and recovery, resulting in improved therapeutic efficacy of allogeneic blood transfusion

\section{Materials and Methods}

This study was an experimental study conducted from the month of June to July 2014.

\subsection{Ethical Consideration}

Ethical clearance for this research was sought from the Ethics and Protocol Review Committee at the School of Biomedical and Allied Health Sciences, University of Ghana, Legon. All the participants gave their informed consent before their samples were collected.

\subsection{Sample Collection}

The samples were collected from the Accra Area Blood Collection site of the Southern Area Blood Centre 
(SABC) located within the premises of Korle-bu Teaching Hospital (KBTH), Accra. Sample analysis was carried out at the haematology research laboratory of the University of Ghana Medical School (UGMS), College of Health Sciences, Korle-bu. A total of 15 samples from consented individuals were used in the study.

\subsection{Materials}

Material used include the following: Saline of varied concentrations (Sigma-Aldrich), Colorimeter, Light microscope \& Haematocrit (Olympus Life and Material Science, Europa GMBH—Germany), Leishman stain-Preprepared according to manufacturer's protocol, Sigma-Aldrich, Buffer (Sigma-Aldrich), CPDA-1 anticoagulant (Fenwal Inc.- -USA), Centrifuge (Eppendorf 5810R, Rotor number A-4-62), slide rack, test tubes and automatic pipettes (Thomas scientific), were used.

\subsection{Participants}

Participants were between the ages of 18 to 47 years and have met all the criteria required to qualify as blood donors by the National Blood Transfusion Service (NBTS). A total of fifteen (15) apparently healthy participants were enrolled.

\subsection{Sample Collection and Processing}

Whole blood samples were collected from each randomly selected replacement donor into a labeled blood bag containing CPDA-1 as an anticoagulant. The samples were gently mixed to avoid clotting and kept in a vaccine carrier before being taken to the haematology research laboratory for analysis. An aliquot of the samples were analyzed within four hours of collection for determination of Packed Cell Volume (PCV), Osmotic Fragility and Thin Film for red cell general film comment to obtain baseline values (Day 1). The samples were then sent to the blood bank, where they were stored in the blood bank refrigerator. The storage temperature was monitored twice daily and aliquots were taken and analyzed in a weekly ( 7 days) interval for 35 days duration. The osmotic fragility test, PCV, and Leishman staining technique was used to determine changes in red blood cell membrane and morphological integrity.

\subsection{Data Analysis}

Data from the study was analysed using Statistical Package for Social Sciences (SPSS) version 20 statistical software. It was checked for completeness and correctness and was double entered into data sheet. Data cleaning and verification was also done regularly. Data collected was transcribed immediately after each session. Anova test was used to determine whether there was any statistical significance between storage periods (Day: 1, 7, 14, 21, 28 and 35). Post Hoc analysis was used to determine the changes obtained between the chosen duration intervals of sample re-analysis throughout the 35 days period of storage. Other descriptive statistics such as mean, median, standard deviation and graph was also done. A p-value of $<0.05$ was taken as being statically significant.

\section{Results}

The study used fifteen (15) samples from replacement blood donors. The minimum and maximum ages of the participants were 18 and 47 years (Table 1).

It was seen that the mean cell fragility (MCF) increased with the duration of storage. Days1 and 7 recorded the least mean value of 0.45 while Day 35 recorded the highest mean value of $0.56 \pm 0.11$ (Table 2).

The MCFs extrapolated from Figure 1 were statistically significant $(F=6.86 ; p=0.001)$ across the various periods of storage by anova analysis. However post hoc analysis (Bonferroni), showed significant changes between the MCF of Day 1 and Day $35\left(p=0.001\right.$, Mean Diff $\left.=-0.1086667^{*}\right)$ and Day 7 and $35(p=0.001$, Mean Diff $\left.=-0.1020000^{*}\right)($ Table 3$)$.

The PCVs decreased with increased storage duration. Day 1 recorded the highest mean PCV value of $38.47 \pm$ 3.11, while Day 35 recorded the least mean PCV value of $35.80 \pm 3.23$ (Table 4). Even though there were changes in the mean PCV throughout the storage periods, these changes were not statistically significant (F-1.684; $\mathrm{p}=0.147$ which is $>0.05)$ as presented in Table 5 . 


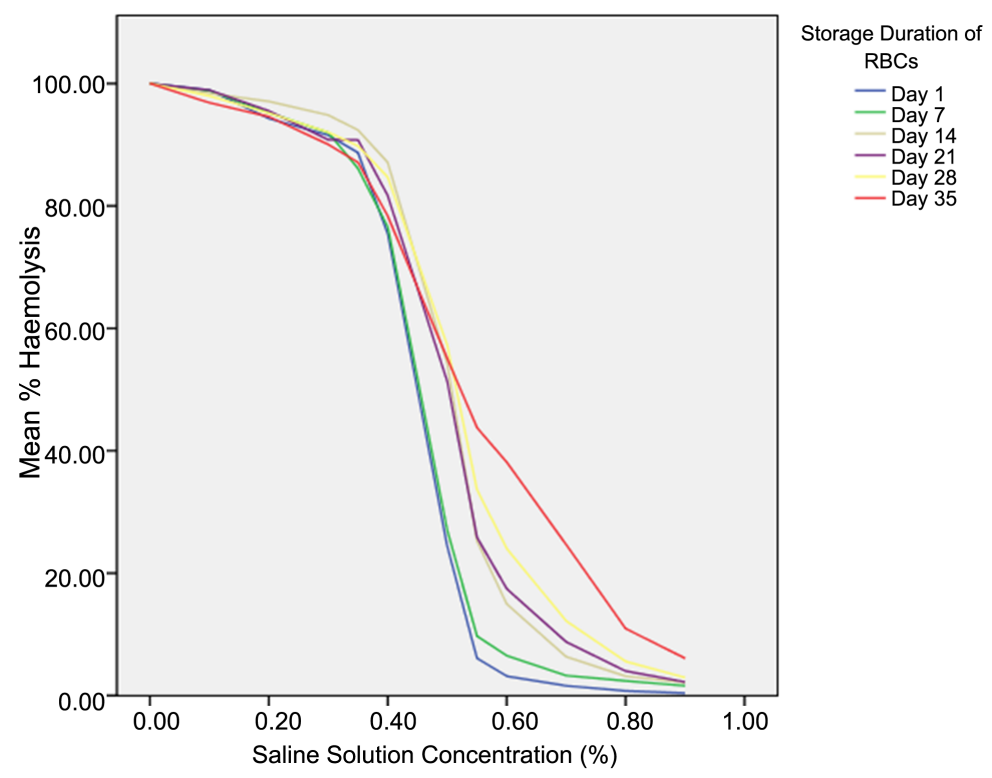

Figure 1. Osmotic fragility curve from which MCFs were extrapolated.

Table 1. Demographic characteristics of participants.

\begin{tabular}{cc}
\hline Variables & Participants \\
\hline Males & $13(86.7 \%)$ \\
Females & $2(13.3 \%)$ \\
Age (Mean) & $(31.87 \pm 6.99)$ \\
Total & $\mathbf{1 5}$ \\
\hline
\end{tabular}

Table 2. Distributions of the mean MCF levels from the various periods of storage.

\begin{tabular}{ccccccccc}
\hline Period of Storage & N & Mean & S.D. & \multicolumn{2}{c}{ 95\% C.I. } & Minimum & Maximum \\
\hline Day 1 & 15 & 0.45 & 0.04 & 0.43 & 0.47 & 0.36 & 0.5 \\
Day 7 & 15 & 0.45 & 0.03 & 0.44 & 0.47 & 0.37 & 0.49 \\
Day 14 & 15 & 0.50 & 0.05 & 0.47 & 0.52 & 0.37 & 0.54 \\
Day 21 & 15 & 0.49 & 0.04 & 0.47 & 0.52 & 0.38 & 0.53 \\
Day 28 & 15 & 0.51 & 0.06 & 0.48 & 0.55 & 0.38 & 0.68 \\
Day 35 & 15 & 0.56 & 0.11 & 0.50 & 0.62 & 0.38 & 0.72 \\
Total & $\mathbf{9 0}$ & $\mathbf{0 . 4 9}$ & $\mathbf{0 . 0 7}$ & $\mathbf{0 . 4 8}$ & $\mathbf{0 . 5 1}$ & $\mathbf{0 . 3 6}$ & $\mathbf{0 . 7 2}$ \\
\hline
\end{tabular}

Table 3. Anova comparison of MCF levels across the various periods of storage.

\begin{tabular}{ccccc}
\hline Duration of Storage & $\mathbf{N}$ & Mean & S.D. & F-Value \\
\hline Day 1 & 15 & 0.45 & 0.04 \\
Day 7 & 15 & 0.45 & 0.03 \\
Day 14 & 15 & 0.50 & 0.05 & 0.682 \\
Day 21 & 15 & 0.49 & 0.04 & $0.001^{* *}$ \\
Day 28 & 15 & 0.51 & 0.06 & 0.11 \\
Day 35 & 15 & 0.56 & & \\
\hline
\end{tabular}

\footnotetext{
${ }^{* *}$ Significant $(\mathrm{p}$-value $<0.05)$.
} 
Table 4. Distribution of mean of PCV across the storage periods.

\begin{tabular}{cccccccc}
\hline Intervention & $\mathbf{N}$ & Mean & Std. Dev & \multicolumn{2}{c}{ 95\% C.I. } & Minimum & Maximum \\
\hline $\mathbf{1}$ & 15 & 38.47 & 3.11 & 36.74 & 40.19 & 33 & 44 \\
$\mathbf{7}$ & 15 & 38.20 & 3.12 & 36.47 & 39.93 & 33 & 44 \\
$\mathbf{1 4}$ & 15 & 37.40 & 2.97 & 35.75 & 39.05 & 32 & 43 \\
$\mathbf{2 1}$ & 15 & 36.80 & 3.10 & 35.08 & 38.52 & 31 & 43 \\
$\mathbf{2 8}$ & 15 & 36.40 & 3.07 & 34.70 & 38.10 & 31 & 42 \\
$\mathbf{3 5}$ & 15 & 35.80 & 3.23 & 34.01 & 37.59 & 30 & 42 \\
Total & $\mathbf{9 0}$ & $\mathbf{3 7 . 1 8}$ & $\mathbf{3 . 1 6}$ & $\mathbf{3 6 . 5 2}$ & $\mathbf{3 7 . 8 4}$ & $\mathbf{3 0}$ & $\mathbf{4 4}$ \\
\hline
\end{tabular}

Table 5. Anova comparison of mean PCV across the storage periods.

\begin{tabular}{ccccc}
\hline Intervention & $\mathbf{N}$ & Mean & Std. Deviation & F-Value \\
\hline $\mathbf{1}$ & 15 & 38.47 & 3.11 & 3.12 \\
$\mathbf{7}$ & 15 & 38.20 & 2.97 & 1.684 \\
$\mathbf{1 4}$ & 15 & 37.40 & 3.10 & $0.147^{*}$ \\
$\mathbf{2 1}$ & 15 & 36.80 & 3.07 & 3.23 \\
$\mathbf{3 5}$ & 15 & 36.40 & $\mathbf{3 . 1 6}$ \\
\hline
\end{tabular}

${ }^{*}$ Not Significant $(\mathrm{p}$-value $>0.05)$.

Photomicrographs from Leishman stained thin films indicate significant degenerative changes in the samples as the storage duration increased. Days 1 and 7 showed generally normocytic normochromic films, Day 14 showed fairly normochromatic with scanty echinocytic cells on the film. Day 21 showed few sphero-echinocytic cells with days 28 and 35 showing hypochromasia with rouleaux formation and marked sphero-echinocytic cells (Figure 2).

\section{Discussion}

In order to achieve efficient therapeutic red cell transfusion with decreased risk of transfusion-associated reactions or events, the concept of blood storage merits scrutiny. This study, revealed that donor blood stored in a preservative (CPDA-1), at the prevailing blood bank conditions over a 35 day period at a temperature of $2^{\circ} \mathrm{C}-$ $8^{\circ} \mathrm{C}$, produced significant changes in erythrocytes osmotic fragility, morphological integrity and marginally decreased changes in PCV.

Results obtained from osmotic fragility test, showed increased MCFs as storage duration progressed from day 1 to 35 as shown in Table 2. Also, significant changes were recorded on day 35 as against day 1. Osmotic fragility test suggests that, as saline concentration increases, physiologically, few "normal" RBCs should be haemolysing unless RBC membrane becomes compromised. In this study, a saline concentration as high as $51 \%$, caused as much as $50 \%$ of the RBCs stored for 28 days to be haemolysed. This was indicative of membrane instability due to the fact that during storage, metabolism activities slow down resulting in decreased ATP and $\mathrm{pH}$ concentrations as discussed by Tinmouth and Chin-Yee [8]. Decreased $\mathrm{pH}$ in RBC metabolic activity inhibits the production of 2, 3-DPG, leading to shape change as discussed elsewhere [9]. The biconcave disk first develops a knobbed appearance and progress into blunt echinocytic projections resulting in ultimate haemolysis as discussed by Shohe and Haley [10].

The trend observed in the study with respect to the RBCs membrane stability is consistent with studies by Tinmouth and Chin-Yee (2001) which showed that, during RBCs storage, metabolism slows down and, as RBCs 
Day 1

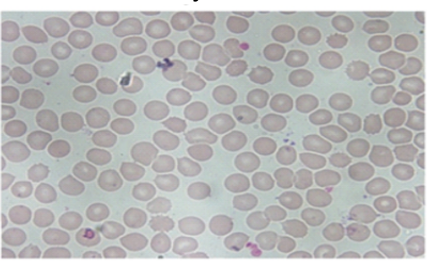

Day 14

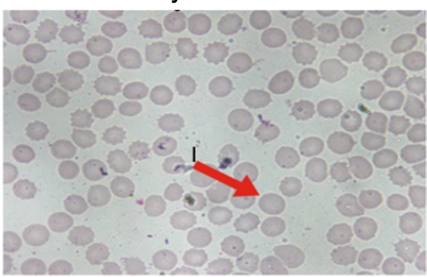

Day 28

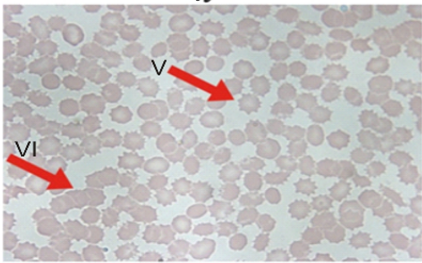

Day 7

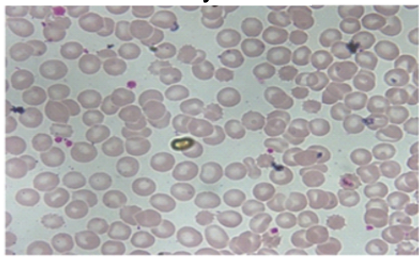

Day 21

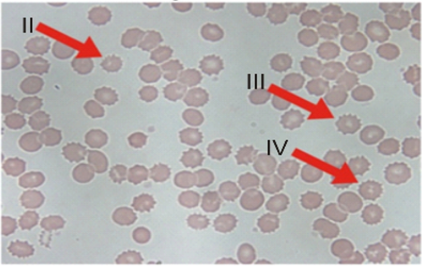

Day 35

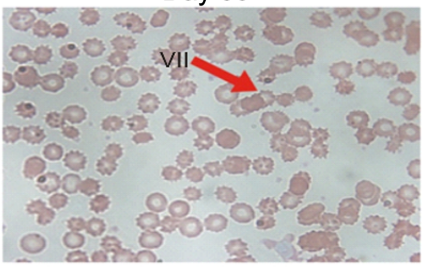

Figure 2. Photomicrograph of representative slides showing morphological abnormities upon storage. Keys: I: Spherocytic Cell. II, III, IV: Echinocytic Cells. VI \& VII: Rouleaux Formed Cells.

"sit" on the refrigerator shelf, metabolic by-products and cellular breakdown products accumulate in the enclosed system. This results in RBC shape change, haemolysis and loss of viability.

From the morphological findings, the film analysis on day 1 to 21 indicated varying but generally non-significant morphological changes (Normocytic Normochromic) as compared to the marked morphological changes-(marked sphero-echnocytic with increased rouleaux formation) seen on films from days 28 and 35. These findings were consistent with our earlier study which posited that, EDTA anticoagulated blood samples stored over time could lead to marked changes in erythrocytes morphology as well as their osmotic fragility, as discussed by Antwi-Baffour et al. [11]. Prolonged storage also leads to increased potassium and free haemoglobin concentration in the suspending plasma, resulting in a fall in $\mathrm{pH}$. At the same time, the cells develop bumps, followed by spicules from which microvesicle bleb, and the cells finally haemolyse. The overall effect leads to decreased fraction of RBCs that survives after being returned to circulation through transfusion as discussed elsewhere [12] [13].

A limitation to this study was the fact that experiments were done in-vitro as compared with an in-vivo study where the stored RBCs would have been radio labeled and returned to circulation before measurements are taken. In an experiment by Hess and Thomas (2003), RBCs stored for six weeks had a mean recovery of $77 \%$ after 24 hours, when transfused into the same donor: This was described as an acceptable measure to determine RBCs functional ability upon prolonged storage. However, although the early stage of the process of RBC storage lesions may be reversible by metabolic rejuvenation of lost components like ATP, 2, 3-DPG and pH, the advanced stage characterized by shedding of membrane cannot be undone irrespective of the preservative used as discussed by Hess [9].

\section{Conclusion}

CPDA-1 is a universally accepted combined anticoagulant-nutrition solution capable of preserving blood for a maximum duration of 35 days. However the findings of this study showed progressive loss of membrane seen as increasing MCF as storage duration increased with most significant change seen on day 35 . The authors there- 
fore conclude that donor blood units stored for 28 days and beyond in CPDA-1 is likely to cause RBC defects that can affect their viability and may prevent practitioners from achieving the expected therapeutic benefit. Rather, it may increase patients risk to transfusion-associated adverse effects.

\section{Acknowledgements}

We are grateful to the management and staff of the Accra Area Blood Collection site of the Southern Area Blood Centre (SABC), Korle-Bu Teaching Hospital, Accra. Also, the haematology research laboratory of the University of Ghana Medical School (UGMS), College of Health Sciences, Korle-Bu.

\section{Competing Interests}

We the author(s) declare(s) that there is no conflict of interest regarding the publication of this paper.

\section{References}

[1] Chessbrough, M. (2010) Medical Laboratory Practice in Tropical Medicine. Cambridge University Press, Cambridge, UK, Vol. 2, 272-273.

[2] United States for Health and Human Services (USDHHS), 2012.

[3] Hess, J.R. and Thomas, M.J.G. (2003) Blood Use in War and Disaster: Lesson from the Last Century. Transfusion, 43, 1622-1633. http://dx.doi.org/10.1046/j.1537-2995.2003.00576.x

[4] Ministry of Health (2007) National Blood Policy for the Health Sector. MoH, Accra.

[5] Daryl, J.K., Camille, M.V.B. and Ognjen, G. (2009) Red Blood Cell Storage Lesion. Bosnia Journal of Basic Medical Science, 9, 26-27.

[6] Copper, G.M. (2000) The Cell: A Molecular Approach. In: Meyer, R., Ed., Structure of the Plasma Membrane, 2nd Edition, Sinauer Associates, Sunderland, MA, USA, 321-235.

[7] Gupta, P.K. (2008) Cell and Molecular Biology. In: Rastogi, R., Ed., The Cell Membrane, 4th Edition, Rastogi Publication, New Delhi, 113-116.

[8] Tinmouth, A. and Chin-Yeem I. (2001) The Clinical Consequences of the Red Cell Storage Lesion. Transfusion Medicine Reviews, 15, 91-107. http://dx.doi.org/10.1053/tmrv.2001.22613

[9] Hess, J.R., Ginine, M.B., et al. (2013) Blood Banking and Transfusion Medicine; Basic Principles and Practice. 2nd Edition, Churchill Livingston, Philadelphia, 205-210.

[10] Shohet, S.B. and Haley, J.E. (1973) Red Cell Membrane Shape and Stability: Relation to Cell Lipid Renewal Pathways and Cell ATP. In: Bessis, M., Weeds, R.I. and Leblond, P.F., Eds., Red Cell Shape: Physiology, Pathology, Ultrastructure, Springer Verlag Inc., New York, 41-48. http://dx.doi.org/10.1007/978-3-642-88062-9 6

[11] Antwi-Baffour, S., Quao, E., Kyeremeh, R. and Mahmood, S.A. (2013) Prolong Storage of Blood in EDTA Has an Effect on the Morphology and Osmotic Fragility of Erythrocytes. International Journal of Biomedical Sciences, 1, 20-23. http://dx.doi.org/10.11648/j.ijbse.20130102.11

[12] Campanella, M.E., Chu, H. and Low, P.S. (2005) Assembly and Regulation of Glycolytic Enzyme Complex on the Human Erythrocyte Membrane. Proceedings of the National Academy of Sciences of the United States of America, 102, 2402-2407. http://dx.doi.org/10.1073/pnas.0409741102

[13] Hess, J.R. (2004) Update on Alternative Oxygen Carriers. Vox Sanguinis, 87, 132-135. http://dx.doi.org/10.1111/j.1741-6892.2004.00469.x 